\title{
Functorial Language Games for Question Answering
}

\author{
Giovanni de Felice ${ }^{\dagger}$, Elena Di Lavore ${ }^{\star}$, Mario Román ${ }^{\star}$, Alexis Toumi ${ }^{\dagger}$ \\ $\dagger$ Department of Computer Science, University of Oxford. \\ $\star$ Department of Software Science, Tallinn University of Technology.
}

\begin{abstract}
We present some categorical investigations into Wittgenstein's language-games, with applications to game-theoretic pragmatics and question-answering in natural language processing.
\end{abstract}

\section{Introduction}

In his 1953 Philosophical Investigations [31], Wittgenstein introduces the concept of language-game (Sprachspiel) as a basis for his theory of meaning. He never gives a general definition, and instead proceeds by enumeration of examples: "asking, thanking, cursing, greeting, praying". Thus, depending on the language-game in which it is played, the same utterance "Water!" can be the answer to a question, a request to a waiter or the chorus of a song. This has often been summarised by the slogan "meaning is use", which became the object of a subfield of linguistics: pragmatics. Since Lewis' work on language conventions [21], formal game theory has been used to model speaker's and hearer's actions in the context of a discourse [1]. In parallel, game theory has been proven significant in designing machine learning tasks [13] and is beginning to be applied to natural language processing [29, 30].

Category theory has been used to formalise both language and games. On the one hand, the distributional compositional (DisCo) models of Coecke et al. [4, 5] define language meaning as a functor from Lambek's pregroup grammars [19] to compact-closed categories such as the category of vector spaces and linear maps. On the other hand, Ghani et al. [11] introduced a monoidal category of open games as a compositional framework for game theory. In [17], Hedges and Lewis proposed to construct language games as functors from grammar to open games. However, their construction required to depart from the pregroup formalism and relied on an open conjecture.

This paper presents some categorical investigations into language games, with applications to gametheoretic pragmatics. In the first two sections, we give an abstract definition of question-answering as an open game, then define pregroup grammars and DisCo models. The proposal of Hedges and Lewis is reformulated within the pregroup formalism, using the free completion of open games as a rigid monoidal category. Two examples of functorial language games are presented: orders and questions, modelling the pragmatics of imperative and interrogative syntax. The abstract question-answering game of the first section is instantiated with respect to a pregroup grammar: the teacher's moves are grammatical questions, the student's strategies are DisCo models. We characterise the Nash equilibria and identify sufficient conditions for the student to succeed. We conclude with a discussion of the link between open games and learning algorithms for natural language processing.

Elena Di Lavore and Mario Román were supported by the European Union through the ESF funded Estonian IT Academy research measure (project 2014-2020.4.05.19-0001).

David I. Spivak and Jamie Vicary (Eds.): Applied Category Theory 2020 (ACT2020)

EPTCS 333, 2021, pp. 311-321 doi 10.4204/EPTCS.333.21
(C) de Felice, Di Lavore, Román, Toumi

This work is licensed under the Creative Commons Attribution License. 


\section{Q\&A as an open game}

Open games [11] are building blocks that can be composed together to construct games in the sense of economic game theory. They provide a compositional description of game theory and a graphical syntax that facilitates reasoning. They express the equilibria of composite games in terms of their components, which might be more tractable.

Definition 1.1 ([11, Definition 3]). Let $X, S, Y, R \in \operatorname{Set}$. An open game $\mathscr{G}:\left(\begin{array}{c}X \\ S\end{array}\right) \stackrel{\sum}{\rightarrow}\left(\begin{array}{l}Y \\ R\end{array}\right)$ that takes observations on the set $X$, produces moves on the set $Y$, receives utilities on the set $R$, and returns coutilities on the set $S$, is a quadruple $\left(\Sigma_{\mathscr{G}}, \pi_{\mathscr{G}}, \kappa_{\mathscr{G}}, E_{\mathscr{G}}\right)$ where:

- $\Sigma_{\mathscr{G}}$ is the set of strategy profiles;

- $\pi_{\mathscr{G}}: \Sigma_{\mathscr{G}} \times X \rightarrow Y$ is the play function, representing how the player following a strategy profile produces a move given an observation;

- $\kappa_{\mathscr{G}}: \Sigma_{\mathscr{G}} \times X \times R \rightarrow S$ is the coplay function, representing how payoffs are propagated from the player to its environment;

- $E_{\mathscr{G}}: X \times(Y \rightarrow R) \rightarrow \mathscr{P}\left(\Sigma_{\mathscr{G}}\right)$ is the equilibrium function, representing the subset of strategies that are best responses for the player in a given context, which consists of a past observation of type $X$ and a continuation of type $Y \rightarrow R$.

Open games form a teleological symmetric monoidal category denoted Game, which admits a graphical calculus developed in [15]. Each morphism is represented as a box with covariant wires for observations and moves, and contravariant wires for utilities and coutilities (see Diagram 11). Closed games $\mathscr{G}: I \rightarrow I$ are determined by a set of strategy profiles $\Sigma_{\mathscr{G}}$ together with an equilibrium function $E_{\mathscr{G}}: 1 \rightarrow \mathscr{P}\left(\Sigma_{\mathscr{G}}\right)$ describing a subset of equilibria. When considering games where players maximise their own utility, these equilibria are precisely Nash equilibria [11, Theorem 2]. Every simultaneous move game, in the sense of classical game theory, defines a closed game [11, Section VII]. Finally, every pair of play/coplay functions can be lifted into a game by picking the trivial strategy set $\mathbf{S}_{\mathscr{G}}=1$ and a constantly true equilibrium function. Open games obtained in this way are called strategically trivial.

Example 1.2. Consider an agent $q$ that poses questions, modelled as a state $q:\left(\begin{array}{l}1 \\ 1\end{array}\right) \nrightarrow\left(\begin{array}{l}Q \\ U\end{array}\right)$ with a set of strategies given by $Q$. We pick some questions-answer pairs $T \subseteq Q \times U$ that the agent finds satisfactory, and these preferences are modelled by defining its equilibrium function to be

$$
E_{\mathscr{G}}(f):=\{q \mid(q, f(q)) \in T\} .
$$

It confronts some oracle that answers the questions, modelled as a strategically trivial effect $a:\left(\begin{array}{l}Q \\ U\end{array}\right) \nrightarrow$ $\left(\begin{array}{l}1 \\ 1\end{array}\right)$. Composing both gives a simple game where the agent tries to ask a question and receive a satisfactory answer. The Nash equilibria of the composite game are precisely the questions that the oracle answers satisfactorily.

Generalizing from this example, we can consider an abstract notion of utility-maximising player.

Definition 1.3. A utility-maximising player with observations in $X$, moves in $Y$, utilities in a partially ordered set $R$, and some subset of possible strategies $\Sigma \subseteq Y^{X}$, is an open game $\mathscr{G}:\left(\begin{array}{c}X \\ 1\end{array}\right) \stackrel{\sum}{\nrightarrow}\left(\begin{array}{l}Y \\ R\end{array}\right)$ with $\pi_{\mathscr{G}}(\sigma, x):=\sigma(x)$ the evaluation function, trivial coplay function; and $E_{\mathscr{G}}(x, \kappa):=\operatorname{argmax}_{\sigma \in \Sigma} \kappa(\sigma(x))$ an equilibrium function describing the subset of the strategies that maximises the utility of the agent.

Let us fix three sets $C, Q, A$ for corpora (i.e. lists of facts), questions and answers respectively. Let $U$ be a set of utilities, which can be taken to be $\mathbb{R}$ or $\mathbb{B}$. We now define a closed game modelling an interaction between three agents: a teacher, a student and a marker. For the moment we define it in terms of abstract sets and unspecified strategies, we will instantiate these in Section 5 . 
Example 1.4 (Teacher, student, marker). A teacher $\mathscr{T}:\left(\begin{array}{l}C \\ 1\end{array}\right) \nrightarrow\left(\begin{array}{c}Q \times A \\ U\end{array}\right)$ is a utility-maximising player where each strategy represents a function turning facts from the corpus into pairs of questions and answers. A student $\mathscr{S}:\left(\begin{array}{l}Q \\ 1\end{array}\right) \nrightarrow\left(\begin{array}{l}A \\ U\end{array}\right)$ is a utility-maximising player where each startegy represents a way of turning questions into answers. A marker is a strategically trivial open game $\mathscr{M}:\left(\begin{array}{l}A \times A \\ U \times U\end{array}\right) \nrightarrow\left(\begin{array}{l}1 \\ 1\end{array}\right)$ with trivial play function and a coplay function defined as $\kappa_{\mathscr{M}}\left(a_{\mathscr{T}}, a_{\mathscr{S}}\right)=\left(-d\left(a_{\mathscr{S}}, a_{\mathscr{T}}\right), d\left(a_{\mathscr{S}}, a_{\mathscr{T}}\right)\right)$ where $d: A \times A \rightarrow U$ is a given metric on A. Finally, we model a corpus as a strategically trivial open game $f:\left(\begin{array}{l}1 \\ 1\end{array}\right) \nrightarrow\left(\begin{array}{l}C \\ 1\end{array}\right)$ with play function given by $\pi_{f}(*)=f \in C$. All these open games are composed to obtain a question answering game in the following way.

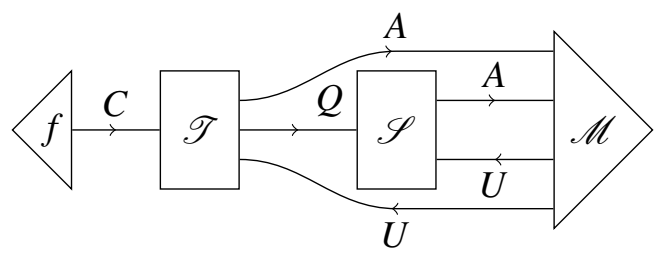

Intuitively, the teacher produces a question from the corpus and gives it to the student who uses his strategy to answer. The marker will receive the correct answer from the teacher together with the answer that the student produced, and output two utilities. The utility of the teacher will be the distance between the student's answer and the correct answer; the utility of the student will be the exact opposite of this quantity. In this sense, question answering is a zero-sum game.

\section{Pregroup semantics}

Pregroup grammars are algebraic models of natural language syntax, first introduced by Lambek [19]. They are weakly equivalent to context-free grammars [3], can be parsed efficiently [23], and can be given functorial semantics [5, 26]. In this section we give background on pregroups and their models in rigid monoidal categories.

Definition 2.1. A preordered monoid is a preorder $P$ equipped with a monotone monoid, i.e. $a \leq c \wedge$ $b \leq d \Longrightarrow a b \leq c d$ for all $a, b, c, d \in P$. A pregroup is a preordered monoid $P$ where every type $t \in P$ has left and right adjoints $t^{l}, t^{r}$ such that $t^{l} t \leq 1 \leq t t^{l}$ and $t t^{r} \leq 1 \leq t^{r} t$.

Definition 2.2. A pregroup grammar is a tuple $G=(V, B, D, s)$ where $V$ is a set of words called the vocabulary, $B$ is a finite set of basic types with $s \in B$ the sentence type, and $D \subseteq V \times P_{B}$ is a finite set of dictionary entries for $P_{B}$ the free pregroup generated by $B$.

A pregroup grammar $G$ generates a language $\mathscr{L}(G, s) \subseteq V^{*}$ as follows. A list of words $u \in V^{*}$ is grammatical, i.e. $u \in \mathscr{L}(G, s)$, whenever for each word $u_{i} \in V, i \leq|u|$ there is a type $t_{i} \in P_{B}$ such that $\left(u_{i}, t_{i}\right) \in D$ and $t_{1} \ldots t_{n} \leq s$ in the free pregroup.

In [24], Lambek and Preller recast the pregroup formalism in terms of free compact 2-categories. We focus on the case of compact 2-categories with one-object, i.e. rigid categories. Let Cat, MonCat and RigidCat denote the categories of categories and functors, monoidal categories and monoidal functors and rigid categories and rigid functors, respectively. Given a set of generating objects $O$, a simple signature over $O$ is a graph $\Gamma \rightrightarrows O$, it generates the free category $\mathbf{C}(\Gamma) \in \mathbf{C a t},[27,2.3]$. A monoidal signature over $O$ is a graph $\Gamma \rightrightarrows O^{*}$ and it generates the free monoidal category $\mathbf{M C}(\Gamma) \in$ MonCat, [27, 3.3]. Finally a rigid signature over $O$ is a graph $\Gamma \rightrightarrows P_{O}$ where $P_{O}$ is the free pregroup on $O, \Gamma$ generates the free rigid category $\mathbf{R C}(\Gamma) \in$ RigidCat, [27, 4.8]. 
For a pregroup grammar $G=(V, B, D, s)$, let $\mathbf{G}:=\mathbf{R C}(D \rightrightarrows B+V)$ be the free rigid category generated by the dictionary entries, where the two maps are given by projections, i.e. $\operatorname{dom}(w, t)=w$ and $\operatorname{cod}(w, t)=t$ for $(w, t) \in D$. The grammatical structure of a sentence $u \in \mathscr{L}(G, s)$ may now be given explicitly by a diagram $g: u \rightarrow s$ in G. In general, for any basic type $b \in B$ we will write:

$$
\mathscr{L}(G, b)=\coprod_{u \in V^{*}} \mathbf{G}(u, b)
$$

Example 2.3. Take $G=(V, B, D, s)$ with $V=\{$ sense, makes, this, sentence $\}, B=\{s, n, d\}$ and $D=$ $\left\{(\right.$ sense,$n),\left(\right.$ makes,$\left.n^{r} s n^{l}\right),($ this,$d),\left(\right.$ sentence,$\left.\left.d^{l} n\right)\right\}$. The following string diagram is a proof that $u=$ "This sentence makes sense" $\in \mathscr{L}(G, s)$ is a grammatical sentence. We do not draw the wires for words and depict the dictionary entries as triangles.

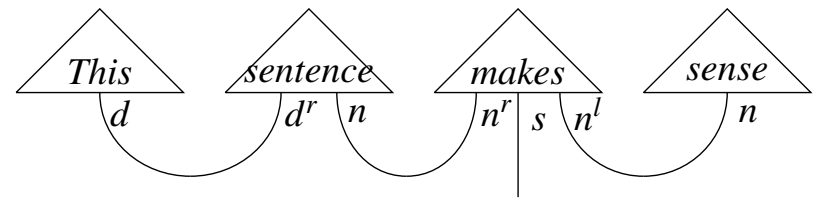

Going from inequalities in a preordered monoid to arrows in a monoidal category allows both to reason about syntactic ambiguity (e.g. "men and (women who run)" vs "(men and women) who run") as well as to define pregroup semantics as a monoidal functor. This second observation lead to the development of the DisCo (distributional compositional) framework [4, 5].

Definition 2.4. A DisCo model for a pregroup grammar $G=(V, B, D, s)$ is a rigid monoidal functor $F: \mathbf{G} \rightarrow \mathbf{S}$ for $\mathbf{S}$ a rigid monoidal category, such that words are sent to the unit $F(w)=1$ for all $w \in V$. The semantics of a list of words $u \in V^{*}$ with grammatical structure $g: u \rightarrow t$ is given by the state $F(g)$ : $1 \rightarrow F(t)$.

Example 2.5. Relational models $F: \mathbf{G} \rightarrow$ Rel correspond precisely to relational databases. The basic types $B$ correspond to a set of attributes, with $F(b)$ the set of data values for each $b \in B$. The dictionary $D \subseteq V \times P_{B}$ corresponds to a database schema, and the functor $F$ to an instance of that schema, mapping each entry $(w, t) \in D$ to a relation $F(w) \subseteq F(t)=F\left(b_{0}\right) \times F\left(b_{1}\right) \cdots \times F\left(b_{k}\right)$ where $\left\{b_{i}\right\}$ is the list of basic types in $t$ (note that $F\left(b^{r}\right)=F(b)=F\left(b^{l}\right)$ as $\mathbf{R e l}$ is compact closed). Finally, morphisms in $\mathbf{G}$ (and sentences $u \rightarrow s$ in particular) correspond to conjunctive queries which can be evaluated by applying the functor $F$, see [7] where this correspondence is spelled out in detail.

Example 2.6. Distributional models $F: \mathbf{G} \rightarrow$ Vect $_{\mathbb{R}}$ can be constructed by counting co-occurences of words in a corpus [14]. The image of the noun type $n \in B$ is a vector space where the inner product computes noun-phrase similarity [26]. When applied to question answering tasks, distributional models can be used to compute the distance between a question and its answer [6].

\section{From pregroups to open games with snake removal}

We now aim to give semantics to pregroup grammars in the category of open games. As Game is not a rigid monoidal category, building such an interpretation is more involved than the cases above. A first attempt would be to use the teleological structure of Game to interpret the cups in pregroup diagrams. However, the models obtained in this way would be very limited: the image of words are all states, i.e. they have trivial observations and co-utilities. This motivated Hedges and Lewis [17] to define the notion of a process grammar as a free rigid category where the words are modeled as generators with arbitrary domains. We propose an alternative construction which does not require any change to the pregroup formalism, using the following lemma. 
Lemma 3.1 ([[8]). The forgetful functor RigidCat $\rightarrow$ MonCat has a left adjoint $\mathscr{A}:$ MonCat $\rightarrow$ RigidCat. Furthermore, the embedding functor $\mathbf{C} \hookrightarrow \mathscr{A}(\mathbf{C})$ is strong monoidal and fully-faithful.

The free completion $\mathscr{A}:$ MonCat $\rightarrow$ RigidCat is called autonomisation in [8], given a monoidal category $\mathbf{C}$ it constructs a rigid monoidal category $\mathscr{A}(\mathbf{C})$ by freely adding adjoints to the objects of $\mathbf{C}$ with formal cups and caps witnessing the adjunctions.

In our context, this means that we can give semantics to pregroup grammars in open games by constructing a DisCo model $F: \mathbf{G} \rightarrow \mathscr{A}($ Game) with $F(s) \in$ Game. Then, by fullness of the embedding Game $\hookrightarrow \mathscr{A}($ Game $)$, we get that the image of any grammatical sentence $g: u \rightarrow s$ in $\mathbf{G}$ is a morphism:

$$
F(g) \in \mathscr{A}(\text { Game })(1, F(s)) \simeq \operatorname{Game}(1, F(s)) .
$$

In other words, once a functor of this type is constructed, all the cups and caps from pregroup diagrams will cancel each other via the snake equation, leaving us with a morphism in Game. We now give the main result of this section, which will allow us to build language games functorially from any functional pregroup grammar.

Definition 3.2. A pregroup type $t \in P_{B}$ is functional if it belongs to the context-free grammar $b\left|t^{r} t\right| t t^{l}$ where $b \in B$. A pregroup grammar $G=(B, V, D, s)$ is called functional if each type in the dictionary $t \in D(V)$ is functional.

The restriction to functional types is very common in the literature on categorial grammar. They correspond to the syntactic types of combinatory categorial grammars [28] and of the product-free Lambek calculus [10, 2], as well as the original types used for Montague semantics [22]. Even if Lambek doesn't state this restriction explicitly, all the pregroup types he uses in his book [20] are of this form.

Proposition 3.3. For any functional pregroup grammar $G$, there exists a monoidal signature $\Gamma_{G}$ and a functor $F_{G}: \mathbf{G} \rightarrow \mathbf{R C}\left(\Gamma_{G}\right)$, such that any DisCo model $F: \mathbf{G} \rightarrow \mathscr{A}$ (Game) factors uniquely as $F=$ $\mathscr{A}(J) \circ F_{G}$ for some $J: \mathbf{M C}\left(\Gamma_{G}\right) \rightarrow$ Game.

Example 3.4. Fix a pregroup grammar $G=(B, V, D, s)$ with $B=\{d, n, s\}$ for determinant, noun, sentence types. We use the dictionary:

$$
D=\left\{(\text { the }, d),\left(\text { person }, d^{r} n\right),\left(\text { who }, n^{r} n s^{l} n\right),\left(\text { explains }, n^{r} s\right),\left(\text { knows }, n^{r} s n^{l}\right),\left(\text { rules }, d^{r} n\right)\right\} .
$$

The following is a grammatical sentence.

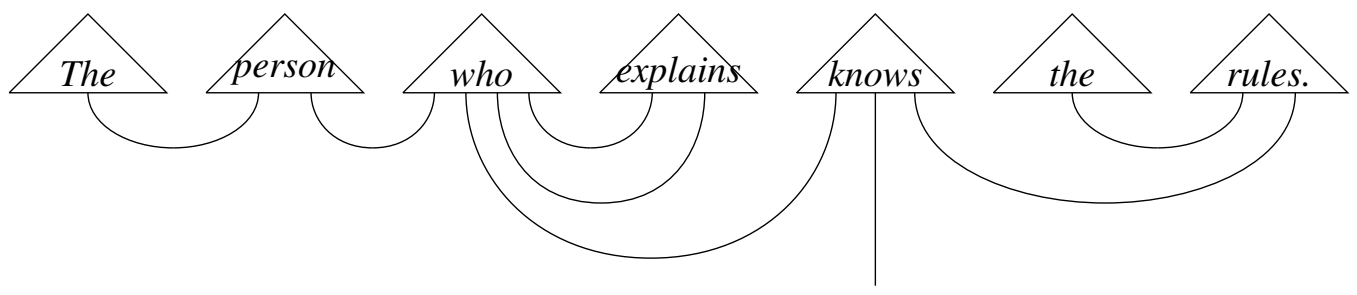

The monoidal signature $\Gamma_{G}$ associated with $G$ is given by: $\Gamma_{G}=\{$ the $: 1 \rightarrow d$; person, rules $: d \rightarrow$ $n$; explains $: n \rightarrow s ;$ knows $: n \otimes n \rightarrow s ;$ who $o_{1}: n \rightarrow a \otimes n ;$ who $\left.2: a \otimes s \rightarrow n\right\}$. The functor $F_{G}$ factors each dictionary entry using cups and the boxes in $\Gamma_{G}$. For instance every entry of the form $\left(w, a^{r} b\right) \in D$ (including adjectives, common nouns and intransitive verbs) and every transitive verb $\left(v, n^{r} n^{l}\right) \in D$ are factored as follows:

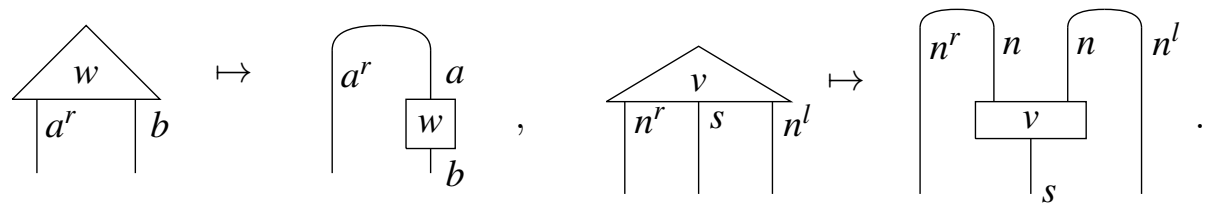


The factorization of "who" requires two boxes:

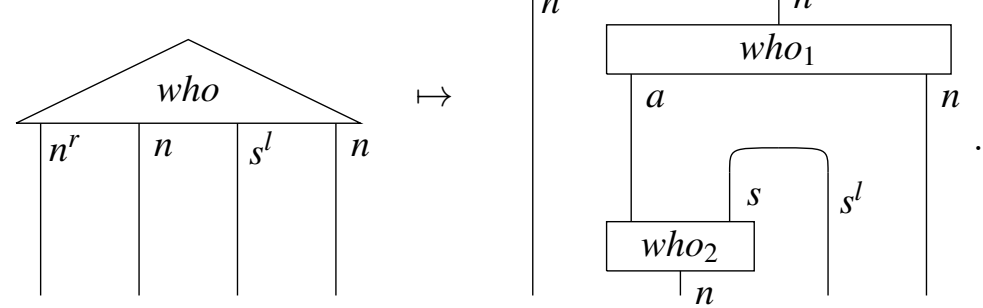

Note that this factorisation carries the same data as a comb in the sense of [18] or equivalently of a lens, see [25] for an account of the relationship between these notions. Applying the functor $F_{G}$ to the sentence (2) and removing the snakes yields the following diagram in $\mathbf{M C}\left(\Gamma_{G}\right) \hookrightarrow \mathbf{R C}\left(\Gamma_{G}\right)$.

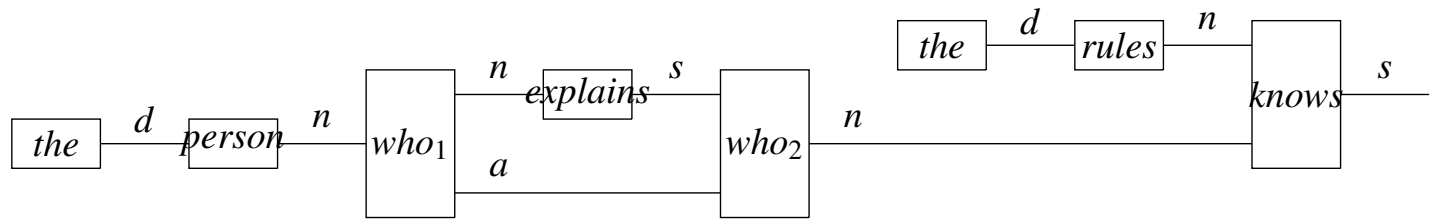

We can now build games for each sentence by finding a functor $J: \mathbf{M C}\left(\Gamma_{G}\right) \rightarrow \mathbf{G a m e}$. In the next section we will use this to obtain a game-theoretic semantics for imperative and interrogative sentences.

\section{Functorial language games}

We start this section by recasting the example of a language game modelled in [17] into our formalism. This is the first example given in the Philosophical Investigations [31] and it features two players: a master builder and his apprentice. The master gives instructions to the apprentice who helps him building with building-stones. They use a language consisting of the following dictionary $D=$ $\left\{\left(\right.\right.$ bring, $\left.s n^{l}\right)$, (large, $\left.n n^{l}\right)$, (slabs, $\left.\left.n\right), \ldots\right\}$, where $n, s \in B$ are the noun and sentence type respectively. The master gives an order to his apprentice by forming a grammatical sentence $u \in O=\mathscr{L}(G, s)$ where $G=(B, V, D, s)$. We can give a game-theoretic semantics to the master's orders as follows. First, the monoidal signature associated with $G$ is $\Gamma_{G}=\{$ bring $: n \rightarrow s$, large $: n \rightarrow n$, slabs $: 1 \rightarrow n\}$, and the functor $F_{G}$ maps "Bring large slabs" as follows:

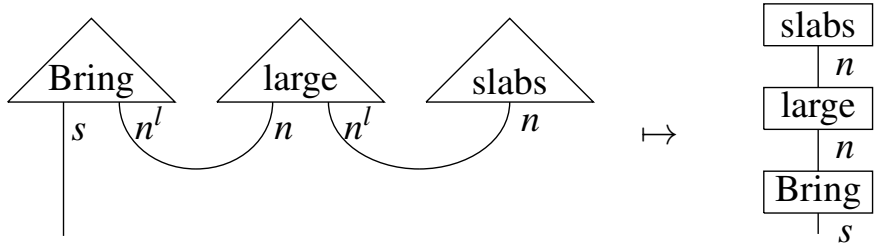

The interpretation as open games $J: \mathbf{M C}\left(\Gamma_{G}\right) \rightarrow$ Game is given by $J(n)=N=\mathscr{L}(G, n)$ and $J(s)=\left(\begin{array}{l}O \\ A\end{array}\right)$ where $A$ is a set of actions with a mapping bring: $N \rightarrow A$, i.e. every noun phrase $x$ refers to some object in the building site and $\operatorname{bring}(x)$ is the action that brings it. We interpret the nouns and adjectives 
syntactically, i.e. $J($ slabs $)=($ slabs $\rightarrow n) \in N$ and

$$
J(\text { large }): N \rightarrow N:: \widehat{x} \mapsto \widehat{x}
$$

The game is encoded in the image of the imperative "Bring", which is interpreted as the open game $J($ bring $):\left(\begin{array}{c}N \\ 1\end{array}\right) \nrightarrow\left(\begin{array}{l}O \\ A\end{array}\right)$ with trivial set of strategy profiles $\Sigma=\star$ and play function: $\pi: \star \times N \rightarrow O: x \mapsto$ $\left(i d_{s} \otimes\right.$ cup $\left._{n}\right) \circ($ Bring $\otimes x)$, analogous to the the image of "large". The coplay function is trivial and the equilibrium $E: N \times(O \rightarrow A) \rightarrow \mathscr{P}(\star)=\mathbb{B}$ is given by:

$$
E(x, k)=\left\{\begin{array}{ll}
1 & k(\pi(x))=\operatorname{bring}(x) \\
0 & \text { otherwise }
\end{array} .\right.
$$

Similar open games can be defined for other orders such as "cut" or "stack". Applying the functor $\llbracket-\rrbracket=$ $\mathscr{A}(J) \circ F_{G}$ to the diagram above, yields an open game $\llbracket$ Bring large slabs $\rrbracket:\left(\begin{array}{l}1 \\ 1\end{array}\right) \nrightarrow\left(\begin{array}{l}O \\ A\end{array}\right)$ with equilibrium function taking a continuation of the apprentice $k: O \rightarrow A$ to:

$$
E_{\llbracket \text { Bring largeslabs } \rrbracket}(k)= \begin{cases}1 & k(\text { Bring large slabs })=\text { bring(large slabs }) \\ 0 & \text { otherwise }\end{cases}
$$

In words, the master is satisfied when his order results in an action that brings large slabs.

Now that we have illustrated the concept of language game with a simple example, we move to a different setup replacing the master-apprentice language with teacher-student pragmatics. We start by modelling the role of a teacher examining his pupil, where orders are replaced by questions. Suppose we are dealing with a philosophy teacher and take the following example:

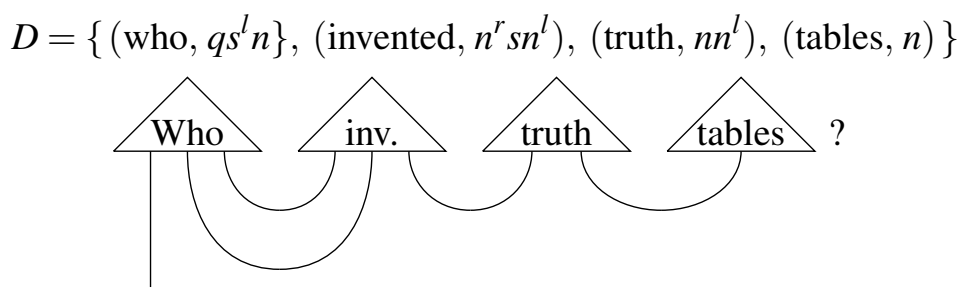

We model the teacher's knowledge of his course with a DisCo model $K_{T}: \mathbf{G} \rightarrow \operatorname{Rel}$ such that $K_{T}(n)=$ $K_{T}(q)=A$ where $A=\{$ Fela, Wittgenstein, Peirce, $\ldots\}$ is a set of names of historical figures. The correct answers to a who-question $g: u \rightarrow q$ are given by a subset $K_{T}(g) \subseteq A$, thus the utility of the student with strategy $k: Q \rightarrow A$ is the intersection $\left\langle k(g) \mid K_{T}(g)\right\rangle \in \mathbb{B}$, see example 2.5 .

As in the previous example, we give a strategically-trivial semantics to each of the words, except for the interrogative "Who" which we use to encode the game. Note that the dictionary entry for "Who" factors via $F_{G}$ as follows:

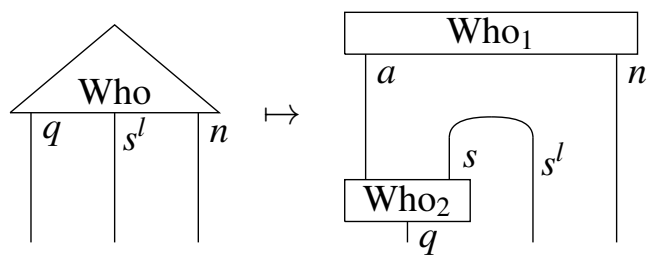


The functor $J: \mathbf{M C}\left(\Gamma_{G}\right) \rightarrow$ Game is defined on objects by $J(a)=J(n)=\left(\begin{array}{c}N \\ 1\end{array}\right)$ and $J(q)=\left(\begin{array}{l}Q \\ A\end{array}\right)$ where $Q=\mathscr{L}(G, q)$ is the set of grammatical who-questions and $N=\mathscr{L}(G, n)$ is the set of grammatical noun phrases. The first part of the factorization initializes the same noun variable $x: 1 \rightarrow n \in \mathbf{G}$ in its two outputs: $\pi_{J\left(\text { Who }_{1}\right)}=(x, x): \star \rightarrow N \times N$, and has trivial coplay function, strategy set and equilibrium. The second play function substitutes the question word "Who" for $x$ in sentence $g$ to build the question:

$$
\pi_{J\left(\mathrm{Who}_{2}\right)}: N \times S \rightarrow Q:(x, g) \mapsto g[x:=\mathrm{Who}] .
$$

It has trivial coplay function and strategy set and an equilibrium $E_{J\left(\mathrm{Who}_{2}\right)}: N \times S \times(Q \rightarrow A) \rightarrow \mathbb{B}$ given by $E_{J\left(\mathrm{Who}_{2}\right)}(x, g, k)=\left\langle k(q) \mid K_{T}(q)\right\rangle$ for $q=\pi_{J\left(\mathrm{Who}_{2}\right)}(x, g)$. As a result, the functor $\llbracket-\rrbracket=\mathscr{A}(J) \circ F_{G}$ maps the question "Who invented truth tables?" to an open game with the following equilibrium function.

$$
E_{\llbracket \text { Who invented truth tables? } \mathbb{}}(k)= \begin{cases}1 & k(\text { Who invented truth tables? }) \in\{\text { Witt., Peirce }\} \\ 0 & \text { otherwise }\end{cases}
$$

In words, the teacher is satisfied when the student answers his question correctly. Note that we could reverse the equilibrium to model a teacher that is satisfied when the student gives the wrong answer. In the next section, we will use this alternative choice to define an adversarial question answering game.

\section{Nash equilibria in a Q\&A game}

In Section 1, we defined an open game for question-answering using abstract sets $C, Q, A$ and $U$ for corpus, questions, answers and utilities. Strategies and plays for the agents were given by arbitrary functions between those sets. We now instantiate those functions with respect to a pregroup grammar $G=(B, V, D, s)$ with a fixed question type $z \in B$. We consider the case where utilities are booleans $U=\mathbb{B}$ and leave the generalisation to any semiring for future work.

We take the corpus $C$ to be a list of question-answer pairs $(q, a)$ for $q: u \rightarrow z$ and $a \in A$. For simplicity, we assume $q$ is a yes/no question and $a$ is a boolean answer, i.e. $Q=\mathscr{L}(G, z)$ and $A=\mathbb{B}$. The strategies of the student are DisCo models $\sigma: \mathbf{G} \rightarrow \operatorname{Rel}$ with $\sigma(z)=1$, so that given a question $q: u \rightarrow z, \sigma(q) \in \mathscr{P}(1)=\mathbb{B}$ is the student's answer. In practice, the student may only have a subset of models available to him so we set $\Sigma_{\mathscr{S}} \subseteq\{\sigma: \mathbf{G} \rightarrow \operatorname{Rel}: \sigma(z)=1\}$. The strategies of the teacher are given by indices $\Sigma_{\mathscr{T}}=\{0,1, \ldots n\}$, so that the play function $\pi_{\mathscr{T}}: \Sigma_{\mathscr{T}} \times(Q \times A)^{*} \rightarrow Q \times A$ picks the question-answer pair indicated by the index. The marker will compare the teacher's answer $a$ with the student's answer $\sigma(q) \in \mathbb{B}$ using the metric $d: A \times A \rightarrow \mathbb{B}::\left(a_{0}, a_{1}\right) \mapsto\left(a_{0}=a_{1}\right)$. Plugging these open games as in Example 1.4, we can compute the set of equilibria of the game by composing the equilibrium functions of its components.

$$
E_{\mathscr{G}}=\left\{(j, \sigma) \in \Sigma_{\mathscr{T}} \times \Sigma_{\mathscr{S}}: j \in \underset{i \in \Sigma_{\mathscr{T}}}{\operatorname{argmax}} a_{i} \neq \sigma\left(q_{i}\right) \wedge \sigma \in \underset{\sigma \in \Sigma_{\mathscr{S}}}{\operatorname{argmax}}\left(a_{j}=\sigma\left(q_{j}\right)\right)\right\}
$$

Therefore, in a Nash equilibrium, the teacher will ask the question that the student, even with his best guess, is going to answer in the worst way. The student, on the other hand, is going to answer as correctly as possible.

We can analyse the possible outcomes of this game.

1. There is a pair $\left(q_{i}, a_{i}\right)$ in $C$ that the student cannot answer correctly, i.e. $\forall \sigma \in \Sigma_{\mathscr{S}}: \sigma\left(q_{i}\right) \neq a_{i}$. Then $i$ is a winning strategy for the teacher and $(i, \sigma)$ is a Nash equilibrium, for any choice of strategy $\sigma$ for the student. If no such pair exists, then we fall into one of the following cases. 
2. The corpus is consistent - i.e. $\exists \sigma: \mathbf{G} \rightarrow$ Rel such that $\forall i \cdot \sigma\left(q_{i}\right)=a_{i}-$ and the student has access to the model $\sigma$ that answers all the possible questions correctly. Then, the strategy profile $(j, \sigma)$ is a Nash equilibrium and a winning strategy for the student for any choice $j$ of the teacher.

3. For any choice $i$ of the teacher, the student has a model $\sigma_{i}$ that answers $q_{i}$ correctly. And viceversa, for any strategy $\sigma$ of the student there is a choice $j$ of the teacher such that $\sigma\left(q_{j}\right) \neq a_{j}$. Then the set $E_{\mathscr{G}}$ is empty, there is no Nash equilibrium.

To illustrate the last case, consider a situation where the corpus $C=\left\{\left(q_{0}, a_{0}\right),\left(q_{1}, a_{1}\right)\right\}$ has only two elements and the student has only two models $\Sigma_{\mathscr{S}}=\left\{\sigma_{0}, \sigma_{1}\right\}$ such that $\sigma_{i}\left(q_{i}\right)=a_{i}$ for $i \in\{0,1\}$ but $\sigma_{0}\left(q_{1}\right) \neq a_{1}$ and $\sigma_{1}\left(q_{0}\right) \neq a_{0}$. Then we're in a matching pennies scenario, both the teacher and the student have no incentive to choose any one of their startegies and there is no Nash equilibrium. This problem can be ruled out if we allowed the players in the game to have mixed strategies, which can be achieved with minor modifications of the open game formalism [12].

\section{Conclusion}

We studied the links between two recently developed applications of category theory: open games and distributional compositional language models. We constructed language games as functors from a pregroup grammar to the free completion of open games as a rigid category and used this construction to give a game-theoretic pragmatics for orders and questions. Finally, we analysed the Nash equilibria of an adversarial Q\&A game. Going towards implementation, the next step is to define a similar Q\&A game in the category of learners of Fong et al. [9], see [16] for the relationship between learners and open games. This would amount to instantiating the strategy sets with the parameters of a learning algorithm, so that gradient descent converges to the desired Nash equilibrium. It would allow to formalise a generative adversarial learning algorithm [13] for question answering.

\section{References}

[1] Anton Benz \& Jon Stevens (2018): Game-Theoretic Approaches to Pragmatics. Annual Review of Linguistics 4(1), pp. 173-191, doi 10.1146/annurev-linguistics-011817-045641

[2] Wojciech Buszkowski (2016): Syntactic Categories and Types: Ajdukiewicz and Modern Categorial Grammars. doi $10.1163 / 9789004311763 \_004$.

[3] Wojciech Buszkowski \& Katarzyna Moroz (2007): Pregroup Grammars and Context-Free Grammars.

[4] Stephen Clark, Bob Coecke \& Mehrnoosh Sadrzadeh (2008): A Compositional Distributional Model of Meaning. In: Proceedings of the Second Symposium on Quantum Interaction (QI-2008), pp. 133-140.

[5] Stephen Clark, Bob Coecke \& Mehrnoosh Sadrzadeh (2010): Mathematical Foundations for a Compositional Distributional Model of Meaning. In J. van Benthem, M. Moortgat \& W. Buszkowski, editors: A Festschrift for Jim Lambek, Linguistic Analysis 36, pp. 345-384.

[6] Bob Coecke, Giovanni de Felice, Dan Marsden \& Alexis Toumi (2018): Towards Compositional Distributional Discourse Analysis. Electronic Proceedings in Theoretical Computer Science 283, pp. 1-12, doi $10.4204 /$ EPTCS.283.1.

[7] Giovanni de Felice, Konstantinos Meichanetzidis \& Alexis Toumi (2019): Functorial Question Answering. arXiv:1905.07408 [cs, math].

[8] Antonin Delpeuch (2017): Autonomization of Monoidal Categories. doi:10.31219/osf.io/efs3b 
[9] Brendan Fong, David Spivak \& Remy Tuyeras (2019): Backprop as Functor: A compositional perspective on supervised learning. In: 2019 34th Annual ACM/IEEE Symposium on Logic in Computer Science (LICS), IEEE, doi:10.1109/lics.2019.8785665.

[10] Timothy A. D. Fowler (2008): Efficiently Parsing with the Product-Free Lambek Calculus. In: Proceedings of the 22nd International Conference on Computational Linguistics (Coling 2008), Coling 2008 Organizing Committee, Manchester, UK, pp. 217-224, doi:10.3115/1599081.1599109.

[11] Neil Ghani, Jules Hedges, Viktor Winschel \& Philipp Zahn (2018): Compositional game theory. In: Proceedings of the 33rd Annual ACM/IEEE Symposium on Logic in Computer Science, pp. 472-481, doi $10.3982 /$ ECTA6297.

[12] Neil Ghani, Clemens Kupke, Alasdair Lambert \& Fredrik Nordvall Forsberg (2019): Compositional Game Theory with Mixed Strategies: Probabilistic Open Games Using a Distributive Law. In: Applied Category Theory Conference 2019.

[13] Ian Goodfellow, Jean Pouget-Abadie, Mehdi Mirza, Bing Xu, David Warde-Farley, Sherjil Ozair, Aaron Courville \& Yoshua Bengio (2014): Generative Adversarial Nets. In Z. Ghahramani, M. Welling, C. Cortes, N. D. Lawrence \& K. Q. Weinberger, editors: Advances in Neural Information Processing Systems 27, Curran Associates, Inc., pp. 2672-2680.

[14] Edward Grefenstette \& Mehrnoosh Sadrzadeh (2011): Experimental Support for a Categorical Compositional Distributional Model of Meaning. In: The 2014 Conference on Empirical Methods on Natural Language Processing., pp. 1394-1404.

[15] Jules Hedges (2017): Coherence for Lenses and Open Games. arXiv:1704.02230 [cs, math].

[16] Jules Hedges (2019): From Open Learners to Open Games. arXiv:1902.08666 [cs, math].

[17] Jules Hedges \& Martha Lewis (2018): Towards Functorial Language-Games. Electronic Proceedings in Theoretical Computer Science 283, pp. 89-102, doi:10.4204/eptcs.283.7.

[18] Aleks Kissinger \& Sander Uijlen (2017): A categorical semantics for causal structure. In: 32nd Annual ACM/IEEE Symposium on Logic in Computer Science, LICS 2017, Reykjavik, Iceland, June 20-23, 2017, IEEE Computer Society, pp. 1-12, doi:10.1109/LICS.2017.8005095

[19] Joachim Lambek (1999): Type Grammar Revisited. In Alain Lecomte, François Lamarche \& Guy Perrier, editors: Logical Aspects of Computational Linguistics, Springer Berlin Heidelberg, Berlin, Heidelberg, pp. 1-27, doi:10.1016/0168-0072(94)00063-9.

[20] Joachim Lambek (2008): From Word to Sentence: A Computational Algebraic Approach to Grammar. Open Access Publications, Polimetrica.

[21] David K. Lewis (1969): Convention: A Philosophical Study. Wiley-Blackwell.

[22] Richard Montague (1970): Universal Grammar. Theoria 36(3), pp. 373-398, doi:10.1111/j.17552567.1970.tb00434.x

[23] Anne Preller (2007): Linear Processing with Pregroups. Studia Logica 87(2-3), pp. 171-197, doi:10.1007/s11225-007-9087-0

[24] Anne Preller \& Joachim Lambek (2007): Free Compact 2-Categories. Mathematical Structures in Computer Science 17(2), pp. 309-340, doi 10.1017/S0960129506005901.

[25] Mario Román (2020): Comb Diagrams for Discrete-Time Feedback. arXiv:2003.06214 [cs].

[26] Mehrnoosh Sadrzadeh, Stephen Clark \& Bob Coecke (2013): The Frobenius Anatomy of Word Meanings I: Subject and Object Relative Pronouns. Journal of Logic and Computation 23(6), pp. 1293-1317, doi $10.1093 / \log$ com/ext044

[27] P. Selinger (2010): A Survey of Graphical Languages for Monoidal Categories. In: New Structures for Physics, Springer Berlin Heidelberg, pp. 289-355, doi:10.1007/978-3-642-12821-9_4.

[28] Mark Steedman (2000): The Syntactic Process. MIT Press, Cambridge, MA, USA. 
[29] Sandeep Subramanian, Sai Rajeswar, Francis Dutil, Chris Pal \& Aaron Courville (2017): Adversarial Generation of Natural Language. In: Proceedings of the 2nd Workshop on Representation Learning for NLP, Association for Computational Linguistics, Vancouver, Canada, pp. 241-251, doi $10.18653 / \mathrm{v} 1 / \mathrm{W} 17-2629$.

[30] Rocco Tripodi \& Roberto Navigli (2019): Game Theory Meets Embeddings: A Unified Framework for Word Sense Disambiguation. In: Proceedings of the 2019 Conference on Empirical Methods in Natural Language Processing and the 9th International Joint Conference on Natural Language Processing (EMNLP-IJCNLP), Association for Computational Linguistics, Hong Kong, China, pp. 88-99, doi:10.18653/v1/D19-1009.

[31] Ludwig Wittgenstein (1953): Philosophical Investigations. Basil Blackwell, Oxford. 